\title{
Realistic Applications for Wireless Sensor Networks
}

\author{
John A. Stankovic, Anthony D. Wood, Tian He
}

\begin{abstract}
Military surveillance, home health care or assisted living, and environmental science are three major application areas for wireless sensor networks. Revolutionary changes are possible in these areas by using wireless sensor networks. To show the breadth and advantages of wireless sensor networks, design and implementation details are presented for three systems, one in each of these three application domains. Key research challenges and the approaches taken to address them are highlighted. Challenges requiring significantly improved solutions are also identified. These systems and others like them provide significant evidence for the utility of wireless sensor networks.
\end{abstract}

\section{Introduction}

Wireless sensor networks (WSN) is an important and exciting new technology with great potential for improving many current applications in medicine, transportation, agriculture, industrial process control, and the military as well as creating new revolutionary systems in areas such as global-scale environmental monitoring, precision agriculture, home and assisted living medical care, smart buildings and cities, and numerous future military applications [29]. In fact, it is difficult to consider any ma-

John A. Stankovic

Anthony D. Wood

University of Virginia

Department of Computer Science

Charlottesville, VA

e-mail: stankovicecs.virginia.edu

Tian $\mathrm{He}$

University of Minnesota

Department of Computer Science and Engineering

Minneapolis, MN

e-mail: tianheecs. umn. edu 
jor application area that cannot benefit from WSN technology. Typically, WSN are composed of large numbers of minimal capacity sensing, computing, and communicating devices and various types of actuators. WSN operate in complex and noisy real world, real-time environments. To date, research and real-world implementations have produced many excellent low level mechanisms and protocols to collect, transport, perform sensor fusion of this raw data and react with control actions. This Chapter discusses three implemented WSN systems which cover important application areas of wireless sensor networks: military surveillance (VigilNet [10, 34]), home medical care (AlarmNet [39]), and environmental science (Luster [27]). One goal of this Chapter is to illustrate key WSN technology as required by diverse application areas. Before describing each of these systems, key overarching research challenges for WSN are briefly presented.

\section{Challenges}

Many challenging research problems must be addressed to produce a realistic WSN application. This section discusses a number of the critical challenges, specifically entitled: from raw data to knowledge, robust system operation, openness and heterogeneity, security, privacy, real-time, and control (actuation). All of these challenges must deal with noisy, uncertain and evolving environments. Each of the following three applications addresses a subset of these challenges appropriate to their purpose.

\subsection{From Raw Data to Knowledge}

Many WSNs can produce vast amounts of raw data. It is necessary to develop techniques that convert this raw data into usable knowledge in an energy efficient manner. For example, in the medical area, raw streams of sensor values must be converted into semantically meaningful activities performed by or about a person such as eating, poor respiration, or exhibiting signs of depression. Main challenges for data interpretation and the formation of knowledge include addressing noisy, physical world data and developing new inference techniques. In addition, the overall system solution must deal with the fact that no inference method is $100 \%$ correct. Consequently, uncertainty in interpreted data can easily cause users not to trust the system. For example, in making decisions it is necessary to minimize the number of false negatives and false positives and guarantee safety, otherwise the system will be dismissed as unreliable. Location (the sensor node or base station) of the data processing is another critical issue: processing at the sensor node consumes energy and is limited by the device capacity, but it saves transmission energy and network contention. The correct tradeoffs on processing location seem system dependent. 


\subsection{Robust System Operation}

Many applications in wireless sensor networks typically initialize themselves by self-organizing after deployment [4]. At the conclusion of the self-organizing stage it is common for the nodes of the WSN to know their locations, have synchronized clocks, know their neighbors, and have a coherent set of parameter settings such as consistent sleep/wake-up schedules, appropriate power levels for communication, and pair-wise security keys [5]. However, over time these conditions can deteriorate. The most common (and simple) example of this deterioration problem is with clock synchronization. Over time, clock drift causes nodes to have different enough times to result in application failures. While it is widely recognized that clock synchronization must re-occur, this principle is much more general. For example, even in static WSN some nodes may be physically moved unexpectedly. More and more nodes may become out of place over time. To make system-wide node locations coherent again, node re-localization needs to occur (albeit at a much slower rate than for clock sync).

These types of required coherence services must combine with many other approaches to produce robust system operation. This includes formal methods to develop reliable code, in-situ debugging techniques [25], on-line fault tolerance [22], in-field-maintenance [2], and general health monitoring services [26]. These problems are exacerbated due to the unattended operation of the system, the need for a long lifetime, the openness of the systems, and the realities of the physical world. The goal is for this collection of solutions to create a robust system [9] in spite of noisy, faulty and non-deterministic underlying physical world realities.

\subsection{Openness and Heterogeneity}

Traditionally, the majority of sensor based systems have been closed systems. For example, cars, airplanes, and ships have had networked sensor systems that operate largely within that vehicle. However, these systems and other WSN systems are expanding rapidly. Cars are automatically transmitting maintenance information and airplanes are sending real-time jet engine information to manufacturers. WSN will enable an even greater cooperation and 2-way control on a wide scale: cars (and aircraft) talking to each other and controlling each other to avoid collisions, humans exchanging data automatically when they meet and this possibly affecting their next actions, and physiological data uploaded to doctors in real-time with real-time feedback from the doctor. WSN require openness to achieve these benefits. However, supporting openness creates many new research problems including dealing with heterogeneity. All of our current composition techniques, analysis techniques, and tools need to be re-thought and developed to account for this openness and heterogeneity. New unified communication interfaces will be required to enable efficient information exchange across diverse systems and nodes. Of course, openness also causes difficulty with security and privacy, the topics of the next two subsections. 
Consequently, openness must provide a correct balance between access to functionality and security and privacy.

\subsection{Security}

A fundamental problem that must be solved in WSN is dealing with security attacks $[3,23,41]$. Security attacks are problematic for WSN because of the minimal capacity devices being used in parts of the systems, the physical accessibility to sensor and actuator devices, and the openness of the systems including the fact that most devices will communicate wirelessly. The security problem is further exacerbated because transient and permanent random failures are commonplace in WSN and failures are vulnerabilities that can be exploited by attackers. However, the considerable redundancy in WSN creates great potential for designing them to continue to provide their specified services even in the face of failures. To meet realistic system requirements that derive from long lived and unattended operation, WSN must be able to continue to operate satisfactorily in the presence of, and to recover effectively from security attacks. The system must also be able to adapt to new attacks unanticipated when the system was first deployed.

\subsection{Privacy}

The ubiquity and interactions of WSN provide many conveniences and useful services for individuals, but also create many opportunities to violate privacy [15]. To solve the privacy problem created by single and interacting WSN of the future, the privacy policies for each (system) domain must be specified. Once specified the WSN system must enforce privacy. Consequently, the system must be able to express users' requests for data access and the system's policies such that the requests can be evaluated against the policies in order to decide if they should be granted or denied. One of the more difficult privacy problems is that systems may interact with other systems, each having their own privacy policies. Consequently, inconsistencies may arise across systems. Once again, on-line consistency checking and notification and resolution schemes are required.

\subsection{Real-Time}

Classical real-time analyses assume a rigorously defined and highly deterministic underlying system model, a set of tasks with known properties, a system that operates in a well controlled environment, and they abstract away from properties of the physical world. For WSN systems, none of these assumptions are true and stream 
models rather than tasks models are prevalent. Further, WSN often support many real-time sensor streams in noisy, uncertain, and open environments. In particular, a very difficult issue is that wireless communication packet delivery is subject to burst losses. New concepts of guarantees must be developed that will likely span a spectrum from deterministic to probabilistic depending on the application, the environment, and noise and interference models [12].

\subsection{Control and Actuation}

Many WSN utilize feedback control theory when actuation is involved. The classical methodology includes creating a model of the system and then deriving a controller using well known techniques to meet stability, overshoot, settling time, and accuracy requirements. A sensitivity analysis is also performed. However, openness and scale create many difficulties for this methodology. Openness means that the model of the system is constantly changing. Human interaction is an integral aspect of openness and this makes modeling extremely difficult. In addition, scaling and interactions across systems also dynamically change the models and create a need for decentralized control. While some work has been performed in topics such as stochastic control, robust control, distributed control, and adaptive control, these areas are not developed well enough to support the degree of openness and dynamics expected in WSN. A new and richer set of techniques and theory is required. It is especially important to understand how large numbers of control loops might interact with each other. To date there have already been examples of WSN where control loops have competed with each other, one indicating an increase in a control variable while the other loop indicating a decrease in the same variable at the same time. Such dependencies must be addressed in real-time and in an adaptive manner to support the expected openness of WSN.

\section{Surveillance Application - VigilNet}

VigilNet [10] is a military wireless sensor network that acquires and verifies information about enemy capabilities and positions of hostile targets. It has been successfully designed, built, demonstrated, and delivered to the Defense Intelligence Agency for realistic deployment. To accomplish different mission objectives, the VigilNet system consists of 40,000 lines of code, supporting multiple existing mote platforms including MICA2DOT, MICA2, and XSM. This section provides detailed technical description of the VigilNet system. 


\subsection{Application Requirements}

The objective of a typical ground surveillance system is to alert the military command to targets of interest, such as moving vehicles and personnel in hostile regions. Such missions often involve a high element of risk for human personnel and require a high degree of stealthiness. Hence, the ability to deploy unmanned surveillance missions, by using wireless sensor networks, is of great practical importance for the military. Successful detection, classification, and tracking require a surveillance system to obtain the current position of a vehicle and its signature with acceptable precision and confidence. When the information is obtained, it has to be reported to a remote base station within an acceptable latency. Several application requirements must be satisfied to make this system useful in realistic environments:

- Longevity: Military surveillance missions typically last from a few days to several months. Due to the confidential nature of the mission and the inaccessibility of the hostile territory, it may not be possible to manually replenish the energy of the power-constrained sensor devices during the course of the mission. In addition, the static nature of the nodes in the field prevents the scavenging of the power from ambient motion or vibration. The small form factor and possible lack of the line of sight (e.g., deployment in the forest) make it difficult to harvest solar power. Hence, the application requires energy-aware schemes that can extend the lifetime of the sensor devices, so that they remain available for the duration of the mission.

- Configuration Flexibility: It is envisioned that VigilNet will be deployed under different densities, topologies, sensing, and communication capabilities. Therefore, it is essential to design an architecture that is flexible enough to accommodate various system scenarios. For example, the system should have an adjustable sensitivity to accommodate different kinds of environment noise and security requirements. In critical missions, a high degree of sensitivity is desired to capture all potential targets even at the expense of possible false alarms. In other cases, it is desired to decrease the sensitivity of the system, maintaining a low probability of false alarms in order to avoid inappropriate actions and unnecessary power dissipation.

- Stealthiness: It is crucial for military surveillance systems to have a very low possibility of being detected and intercepted. Miniaturization makes sensor devices hard to detect physically; however, RF signals can be easily intercepted if sensor devices actively communicate during the surveillance stage. During the surveillance phase, a zero communication exposure is desired in the absence of significant events.

- Real-time: As a real-time online system for target tracking, VigilNet is required to cope with fast changing events in a responsive manner. For example, a sensor node has to detect and classify a fast moving target within a few seconds before the target moves out of the sensing range. The real-time guarantee for sensor networks is more challenging due to the following reasons. First, sensor networks directly interact with the real world, in which the physical events may exhibit un- 


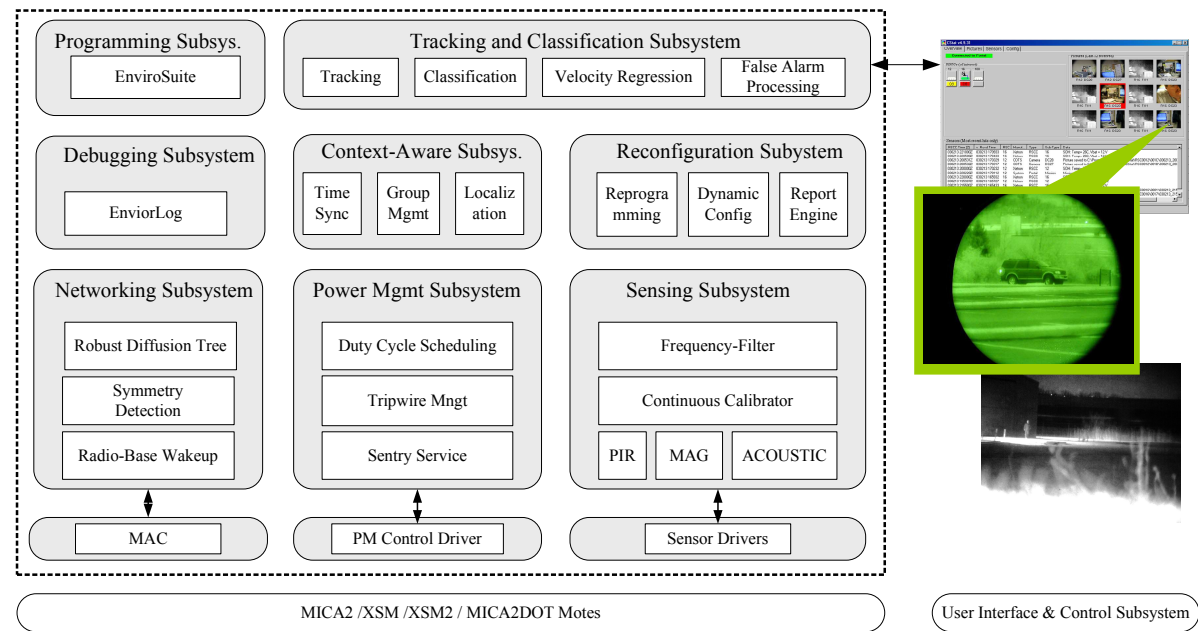

Fig. 1 The VigilNet System Architecture

predictable spatiotemporal properties. These properties are hard to characterize with traditional methods. Second, although the real-time performance is a key concern, it should be performance compatible with many other critical issues such as energy efficiency and system robustness. For example, the delays introduced by power management directly affect the maximum target speed VigilNet can track. It is an essential design tradeoff to balance between network longevity and responsiveness.

\subsection{VigilNet Architecture}

The VigilNet system is designed with a layered architecture as shown in Figure 1. This architecture provides an end-to-end solution for supporting military surveillance applications with multiple essential subsystems.

\subsubsection{Sensing Subsystem}

Sensing is the basis for any surveillance system. The VigilNet sensing subsystem implements detection and classification of targets using continuous online sensor calibration (to a changing environment) and frequency filters to determine critical target features. These filters extract the target signatures from a specific spectrum band, eliminating the burden of applying a computation-intensive Fast Fourier Transform. The sensing subsystem contains three detection algorithms for the magnetic sensor, acoustic sensor, and passive infrared sensor (PIR), respectively. 
- The magnetic sensor detection algorithm computes two moving averages over the most recent magnetic readings. The slower moving average, with more weight on previous readings, establishes a baseline to follow the thermal drift noise caused by the changing temperature during the day. The faster moving average, with more weight on the current reading, detects the swift change in magnetic filed caused by ferrous targets. To make a detection decision, the difference between the two moving average values is compared to a dynamic threshold, which is established during the calibration phase.

- The acoustic sensor detection algorithm uses a lightweight power-based approach. It first computes a moving average of multiple recent acoustic readings, then establishes an auto-adapting acoustic threshold by calculating a moving standard deviation of readings over a certain time window. If an acoustic reading is larger than the sum of the moving average and its corresponding moving standard deviation, it is considered to be a crossover. If the number of crossovers exceeds a certain threshold during a unit of time, this algorithm signals a detection to the upper layer components.

- The passive infrared sensor is designed to sense changes in thermal radiation that are indicative of motion. When there is no movement, the thermal reading is stable and does not trigger detections. If an object is moving in front of a PIR sensor, this object causes a thermal disturbance, triggering the PIR. Most moving objects, such as shaking leaves, rain drops, and vehicles, can trigger the PIR sensor. However, different thermal signatures generate trigger events with different frequencies. Low frequency detections $(<2 \mathrm{~Hz})$ are normally triggered by windinduced motion and other slow moving objects. On the other hand, fast-moving targets such as vehicles generate signals with a much higher frequency. Therefore, it is sufficient to design a high pass ARMA filter to filter out the frequency components lower than $2 \mathrm{~Hz}$. Since in realistic environments temperature and humidity vary significantly over the course of a day, similar to other detection algorithms, PIR's detection threshold is dynamically adjusted to accommodate such environmental changes.

\subsubsection{Context-Awareness Subsystem}

Sensed data is meaningful only when it is interpreted along with the context in which it is obtained. For example, a temperature reading is useless, if it is not associated with a location and time that the value is measured. The context-awareness subsystem comprises lower-level context detection components such as time synchronization and localization. These components form the basis for implementing other subsystems, such as the tracking subsystem. Localization ensures that each node is aware of its location, in order to determine the location of detected targets. Time synchronization is responsible for synchronizing the local clocks of nodes with the clock of the base station, so that every node in the network has a consistent global view of time. Combining time synchronization and localization, it is feasible to estimate the velocity of targets. 
The VigilNet system uses a variation of the time synchronization protocol developed by Maroti et. al. [21]. This variation eliminates the periodic time adjustments for the sake of stealthiness. To localize sensor nodes, VigilNet uses a walking GPS solution [30], in which the deployer (either person or vehicle) carries a GPS device that periodically broadcasts its location. The sensor nodes being deployed infer their position from the location broadcast by the GPS device.

\subsubsection{Tracking and Classification Subsystem}

When a target is detected by a set of nearby nodes, the tracking component creates a group. All nodes that detect the same event join a tracking group to ensure the uniqueness (one-to-one mapping of external events to logical groups) and consistent identification (immutability of the mapping function) of targets, as long as targets are far enough apart from each other or have different signatures. When targets are very near each other and possess an identical signature, a disambiguation mechanism based on their path-histories is used.

Classification is achieved through a hierarchical structure consisting of four tiers: sensor-level, node-level, group-level, and base-level. The classification result is represented by a data structure called the confidence vector. The confidence vector comprises the confidence levels for specific classes of targets, and is used as a common data structure to transport information between different levels of the classification hierarchy.

- The sensor level deals with individual sensors and comprises the sensing algorithms for the corresponding sensors. With communication being an energy costly operation, the sensing algorithms need to perform local detection and classification as much as possible. After processing the sensor data, each sensing algorithm delivers the confidence vector to the higher level module the node-level detection and classification module.

- The node-level classification deals with output from multiple sensors on the node. The fusion of the data from various sensors exposes more useful information than can be obtained from any individual sensor. Hence, the node-level sensing algorithm must correlate the sensor data from individual sensors and form node-level classification results. Such a correlation can enhance the detection and classification accuracy on individual nodes; and different sensors may strengthen the confidence of each other's classification results and invalidate false positives.

- The group-level classification is performed by groups of nodes. Such groups are managed by a middleware called EnviroSuite [17], which provides a set of distributed group management protocols to dynamically organize nodes in the vicinity of targets into groups and elect leaders among them. These leaders are designated to collect the node-level classification results from individual members and, based on them, perform the group-level classification. Thus, the input to the group-level classification is the node-level confidence vectors rather than a bulk of sample points. This greatly reduces the volume of information transmitted between group leaders and members. 
- The highest level in the hierarchical classification architecture is the base-level classification. The group-level classification results are transported via multiple hops to the base station, serving as the input to the base-level classification algorithm. The base-level classification algorithm finalizes the sensing and classification result, and computes various event attributes (e.g., target velocity).

\subsubsection{Networking Subsystem}

After VigilNet collects detection information about incoming targets through the tracking and classification subsystem, it needs to deliver detection reports back to the control center through a multi-hop network. The networking subsystem consists of three major components: a link symmetry detection service, a robust diffusion service, and a radio-based wakeup service. Low power radio components, such as Chipcon CC1000 used by MICA2 [6], exhibit very irregular communication patterns. To address this problem, a Link Symmetry Detection (LSD) module is used to reduce the impact of radio irregularity on upper layer protocols. The main idea of the LSD module is to build a symmetric overlay on top of the anisotropic radio layer, so that those protocols whose correctness depends on the link symmetry can be used without modification. More details on this solution can be found in [42]. The robust diffusion service utilizes a well-known path-reversal technique. Basically, a base node disseminates tree construction requests to the rest of the network with a running hop-count initialized to zero. Requests are flooded outwards with hop-count incremented at every intermediate hop. After receiving tree construction requests, nodes establish multiple reverse paths towards the sending node. As a result, a multi-parent diffusion tree is constructed with the base node residing at the root. The Radio-based Wakeup service is designed to ensure end-to-end data delivery even if intermediate nodes are in the dormant state (due to power management). To support the illusion of on-demand wakeup, a dormant node wakes up and checks the radio activity periodically (e.g., for five milliseconds every several hundred milliseconds). If no radio activity is detected, this node goes back to sleep. Otherwise, it remains active to receive and relay messages. If an active node wants to wake up all neighboring nodes, it only needs to send out a message with a long enough preamble to last longer than the checking period of the dormant nodes.

\subsubsection{Graphic User Interface and Control Subsystem}

The networking subsystem delivers the reports to one or more command and control centers, where the Graphic User Interface and Control subsystem is located. This subsystem provides three major functionalities. First, it accepts the reports from the sensor field and displays such information graphically to the mission operators. Second, it allows the mission operators to disseminate the system configurations through the reconfiguration subsystem. Third, based on the initial detections from the sensor field, it makes final decisions on whether to wake up more advanced 
sensors. These advanced sensors not only classify the type of targets, but also differentiate the model of the targets. Since they are extremely power consuming, they are normally turned off and only used when awakened by initial detections coming from the sensor field.

\subsubsection{The Power Management Subsystem}

One of the key design objectives of the VigilNet system is to increase the system lifetime to $3 \sim 6$ months in a realistic deployment. Due to the small form factor and low-cost requirements, sensor devices such as XSM motes [7] are equipped with limited power sources (e.g., two AA batteries). The normal lifetime for such a sensor node is about 4 days if it remains active all the time. To bridge such a gap, VigilNet is equipped with a power management subsystem. Among all the middleware services, the tripwire service, sentry selection, duty cycle scheduling, and wakeup service form the basis for the power management subsystem. These services are organized into a multi-dimensional architecture. At the top level, the tripwire service is used to divide the sensor field into multiple sections, called tripwire sections. A tripwire section can be scheduled either into an active or a dormant state at a given point of time. When a tripwire section is dormant, all nodes within this section are in a deep-sleep state to conserve energy. When a tripwire section is active, a second-level sentry service is applied within this section. The basic idea of the sentry service is to select only a subset of nodes, defined as sentries, to be in charge of surveillance. Other nodes, defined as non-sentries, can be put into a deep-sleep state to conserve energy. Rotation is periodically done among all nodes, selecting the nodes with more remaining energy as sentries. At the third-level, since a target can normally be sensed for a non-negligible period of time, it is not necessary to turn sentry nodes on all the time. Instead, a sentry node can be scheduled in and out of sleep state to conserve energy. The sleep/awake schedule of a sentry node can be either independent of other nodes or coordinated with that of others in order to further reduce the detection delay and increase the detection probability. More information on VigilNet power management can be found in [11].

\subsubsection{The Reconfiguration Subsystem}

The VigilNet system is designed to accommodate different node densities, network topologies, sensing and communication capabilities, and different mission objectives. Therefore, it is important to design an architecture that is flexible enough to accommodate various system scenarios. The reconfiguration subsystem addresses this issue through two major components: a multi-hop reconfiguration module and a multi-hop reprogramming module. The reconfiguration module allows fast parameter tuning through a data dissemination service, which supports limited flooding. Data fragmentation and defragmentation are supported in the reconfiguration subsystem to allow various sizes of the system parameters. The reprogramming module 
provides a high level of flexibility by reprogramming the nodes. More information on reprogramming can be found in [13].

\subsubsection{The Debugging Subsystem}

Debugging and tuning event-driven sensor network applications such as VigilNet are difficul for the following reasons: (i) big discrepancies exist between simulations and empirical results due to various practical issues (e.g., radio and sensing irregularity) not captured in simulators, which makes them less accurate; (ii) In-field tests of the system require walking or driving through the field to generate events of interest actively, which makes in-field tests extremely costly. To address this issue, a debugging subsystem called EnviroLog [18] is added into VigilNet. EnviroLog logs environmental events into non-volatile storage on the motes (e.g., the $512 \mathrm{~KB}$ external flash memory) with time-stamps. These events can then be replayed in their original time sequence on demand. EnviroLog reduces experimental overhead by eliminating the need to physically re-generate events of interest hundreds of times for debugging or parameter tuning purposes. It also facilitates comparisons between different evaluated protocols.

\subsection{The Programming Interface}

The programming interface in VigilNet is an extension of the prior work on EnviroSuite [17]. It adopts an object-based programming model that combines logical objects and physical elements in the external environment into the same object space. EnviroSuite differs from traditional object-oriented languages in that its objects may be representatives of physical environmental elements. EnviroSuite makes such objects the basic computation, communication, and actuation unit, as opposed to individual nodes. Thus, it hides implementation details such as individual node activities and interactions among nodes. Using language primitives provided by EnviroSuite, developers of tracking or monitoring applications can simply specify object creation conditions (sensory signatures of targets), object attributes (monitored aggregate properties of targets), and object methods (desired computation, communication or actuation in the vicinity of targets). Such specifications can be translated by an EnviroSuite compiler into real applications that are directly executable on motes. When defined object conditions are met, dynamic object instances are automatically created by the run-time system of EnviroSuite to collect object attributes and execute object methods. Such instances float across the network following the targets they represent, and are destroyed when the targets disappear or move out of the network. 


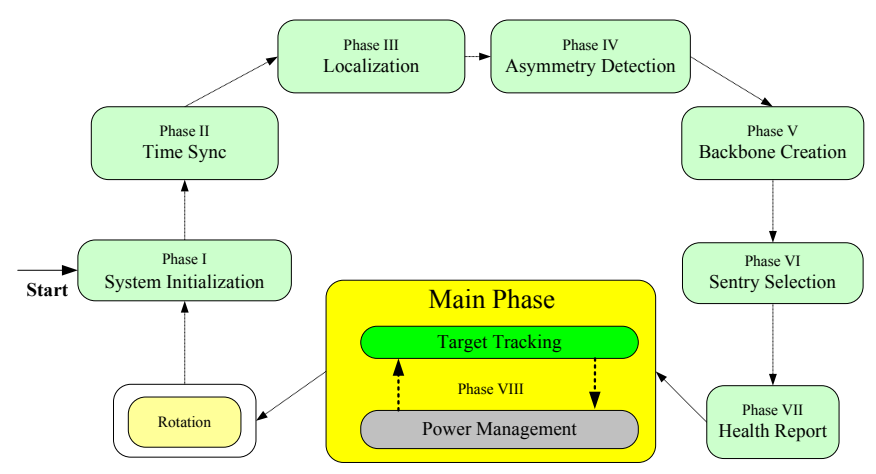

Fig. 2 Phase Transition and Rotation

\subsection{System Work Flow}

To avoid interference among different operations, VigilNet employs a multiplephase work flow. The transition between phases is time-driven, as shown in Figure 2. Phases I through VII comprise the initialization process which normally takes about several minutes. In Phase I, the reconfiguration subsystem initializes the whole network with a set of parameters. In Phase II, the context-awareness subsystem synchronizes all nodes in the field with the master clock at the base, followed by the localization process in Phase III. In Phase IV and V, the networking subsystem establishes a robust diffusion tree for end-to-end data delivery. Phase VI invokes the power management subsystem to activate tripwire sections and select a subset of the nodes as sentries. The system layout, sentry distribution, and network topology are reported to the graphic user interface and control subsystem in Phase VII. After that, the nodes enter into the main phase VIII- the surveillance phase. In this phase, nodes enable the power management subsystem in the absence of significant events, and activate the tracking subsystem once a target enters into the area of interest.

\subsection{VigilNet Summary}

Surveillance using wireless sensor networks is a very practical application. It has many advantages such as fast ad hoc deployment, fine-grained robust sensing and tracking, low-power consumption, and low cost. From the experience in building VigilNet, it is clear that realistic issues must not be ignored in developing usable solutions. This includes sensor failures, environmental changes, asymmetries in communication, and false alarms. Because of these issues, debugging and performance tuning in distributed sensor networks are extremely time consuming, especially during field tests. Therefore, it is critical to have appropriate built-in system support for these functions, such as the reconfiguration subsystem and the debugging subsystem. Since the sensor nodes fail at a much higher rate in hostile outdoor environ- 
ments, self-healing should be supported by every protocol integrated into the system. Despite these realistic challenges faced, VigilNet presents a proof that viable surveillance systems can be implemented and successfully deployed on low-power sensor devices.

\section{Healthcare Applications - AlarmNet}

An aging baby-boom generation is stressing the U. S. healthcare system, causing hospitals and other medical care-givers to look for ways to reduce costs while maintaining quality of care. It is economically and socially beneficial to reduce the burden of disease treatment by enhancing prevention and early detection. This requires a long-term shift from a centralized, expert-driven, crisis-care model to one that permeates personal living spaces and involves informal caregivers, such as family, friends, and community.

Systems for enhancing medical diagnosis and information technology often focus on the clinical environment, and depend on the extensive infrastructure present in traditional healthcare settings. The expense of high-fidelity sensors limits the number that are available for outpatient deployment, and some require specialized training to operate. Manual record keeping has been identified as a key source of medical errors [24], and at its best, traditional data collection is intermittent, leaving gaps in the medical record.

Wireless Sensor Networks (WSNs) provide capabilities that are valuable for continuous, remote monitoring, as research into military and environmental systems attest. For healthcare applications, they can be deployed inexpensively in existing structures without IT infrastructure. Data are collected automatically, enabling daily care and longitudinal medical monitoring and diagnosis. The wireless devices can integrate with a wide variety of environmental and medical sensors.

While addressing some of the needs of distributed healthcare, WSNs also present their own challenges, both practical and theoretical, to being robust platforms for pervasive deployment. Privacy and security of collected medical data may be jeopardized by careless use of a wireless medium. Without smart power management, battery-powered sensors have short lifetimes of a few days or require continual maintenance.

In this section the AlarmNet system is described. AlarmNet is an Assisted-Living and Residential Monitoring Network for pervasive, adaptive healthcare in assistedliving communities with residents or patients with diverse needs. Various parts of AlarmNet have been described before [28, 8, 40, 35, 39], but here the focus is on the following aspects:

- An extensible, heterogeneous network middleware that addresses the challenges of an ad hoc wide-scale deployment, and integrates embedded devices, back-end systems, online analysis, and user interfaces; 
- SenQ, a query protocol for efficiently streaming online sensor data to the system and to users, integrated with privacy, power management, and activity analysis, and

- Novel context-aware protocols using two-way network information flow: environmental, system, and resident data flow into the back-end, and circadian activity rhythm analysis feeds back into the system to enable smart power management and dynamic alert-driven privacy tailored to an individual's activity patterns.

\subsection{AlarmNet Architecture}

A key requirement for healthcare systems is the ability to operate continuously over long time periods and still integrate new technologies as they become available. AlarmNet satisfies these objectives by unifying and accommodating heterogeneous devices in a common architecture (see Figure 3 ) that spans wearable body networks, emplaced wireless sensors, user interfaces, and back-end processing elements.

Fig. 3 Multi-tier AlarmNet architecture with emplaced sensors, mobile body area networks, a backbone of gateways, user interfaces, and back-end storage and processing.

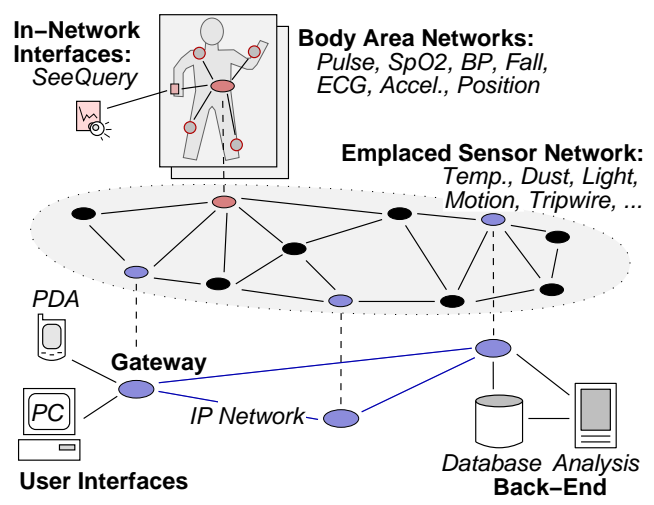

Mobile Body Networks are wireless sensor devices worn by a resident which provide activity classification or physiological sensing, such as an ECG, pulse oximeter, or accelerometers. The network is tailored to the patient's own medical needs, and can provide notifications (for example, alerts to take medicine) using an in-network wearable interface, the SeeMote [28] that has a color LCD.

It also integrates SATIRE [8], a body network that classifies Activities of Daily Living (ADLs) [16] by analyzing accelerometer data generated by a wearer's movements.

Body networks contain a designated gateway device that mediates interaction with the surrounding WSN. This modularizes the system's interaction with the body network to ease its integration. Data are streamed directly or multi-hop through the 
emplaced network to the AlarmGate gateways for storage, analysis, or distribution to user interfaces.

Emplaced Sensors are deployed in living spaces to sense environmental quality, such as temperature, dust, and light, or resident activities. Motion and tripwire sensors, in particular, provide a spatial context for activities and enable location tracking.

Due to their low-cost, small form factor, and limited power budget, the devices answer queries for local data and perform limited processing and caching. Though some deployment environments may enable the use of wired electrical power, it is not required so as to support ad hoc retro-fitting of existing structures. Figure 4(a) shows the lightweight stack resident on sensor devices.

AlarmNet supports dynamically adding new devices to the network, which register their capabilities and are initialized. This flexibility allows the system to change over time as sensors are developed or new pathologies require monitoring.

AlarmGate applications run on an embedded platform, such as the Crossbow stargate, and serve as a communication backbone and application-level gateway between the wireless sensor and IP networks. Owing to their greater resources, these devices perform major aspects system operation related to dynamic privacy, power management, query management, and security. The AlarmGate software stack is shown in Figure 4(b).

Back-end programs perform online analysis of sensor data, feeding back behavior profiles to aid context-aware power management and privacy. A database provides long-term storage of system configuration, user information, privacy policies, and audit records.

One such program, for Circadian Activity Rhythm (CAR) analysis, processes sensor data stored in the database and learns behavior patterns of residents. These are used to detect deviations from personal norms that may signal a short- or longterm decline in resident health.

The back-end is extensible to new analyses using a modular framework, wherein programs consume input sensor streams, filter and process them, and produce output streams in the database for other modules to use. These are composed hierarchically from low-level sensor streams to high-level inference of symptoms and diseases.

User Interfaces allow doctors, nurses, residents, family, and others to query sensor data, subject to enforced privacy policies. We developed a patient-tracking GUI for a nurse's station, and a query issuer for a PDA that graphs sensor data in real-time. These programs are not trusted components-they must connect through AlarmGate and do not have direct access to the database. This makes it easier to develop and deploy new interfaces customized to the application's needs.

In summary, AlarmNet's architecture supports health monitoring applications due to its flexibility and extensibility in 1) supporting dynamic addition of heterogeneous devices, sensors, and body networks, 2) feeding learned resident and system context back into the network, and 3) providing an open client model for future extension. 


\begin{tabular}{|c|c|}
\hline $\begin{array}{c}\text { Query } \\
\text { Processor }\end{array}$ & $\begin{array}{c}\text { Power } \\
\text { Manager }\end{array}$ \\
\hline Sampler & AMSecure \\
\hline Sensor Driver & Routing \\
\hline Sensor HW & Network \\
\hline
\end{tabular}

(a) Embedded software

stack on sensor devices.

\begin{tabular}{|c|c|c|c|}
\hline $\begin{array}{c}\text { Query } \\
\text { Manager }\end{array}$ & $\begin{array}{c}\text { Privacy } \\
\text { Manager }\end{array}$ & $\begin{array}{c}\text { Power } \\
\text { Manager }\end{array}$ & $\begin{array}{l}\text { Database } \\
\text { Interface }\end{array}$ \\
\hline \hline AMSecure & Audit Log & Authentication \\
\hline \hline PhoenixSource & Routing & Client Manager \\
\hline Sensor Network & IP Network \\
\hline
\end{tabular}

(b) AlarmGate software stack on network gateways.

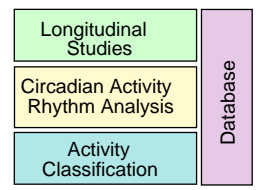

(c) Back-end analysis and persistent storage.

Fig. 4 AlarmNet software architecture.

\subsection{Query Management}

A primary reason for developing AlarmNet was to use environmental, physiological, and activity data of assisted-living residents to improve their health outcomes. The automated analysis programs need to automatically collect data in the background, but the system must also support ad hoc queries by healthcare providers and the addition of new analysis programs over time. Existing data management solutions were mostly optimized for tree-based aggregation [19], or else used general-purpose virtual machines for arbitrary computation.

$\operatorname{Sen} Q$ is a query system that satisfies the requirements of the healthcare domain: reconfigurable in-network sensing and processing, dynamic query origination by embedded devices, and high-level abstractions for expressing queries. A detailed treatment of SenQ's lower layers and their performance was given in [40], with focus on sensing and in-network query processing. Here the system-level query management functions and their integration with other components of AlarmNet are presented.

The back-end system, user interfaces, and embedded devices all issue queries using a common network protocol, in which queries are uniquely identified by $<$ source ID, query ID > tuples. Users may request a snapshot of the current value or a periodic stream of a sensing modality. To reduce repetitive query parsing overhead on resource-constrained motes, both types of queries may be cached and efficiently restarted (or reissued) later.

Since radio communication in the WSN is expensive, it is desirable to process data at its source, if possible, to reduce the amount that is reported. However, sensor devices have limited memories and processors, and so only relatively lightweight processing is practical. SenQ dynamically constructs a scalar processing chain on the mote to perform spatial and temporal aggregation and filtering to reduce the energy consumed by communication.

Query Manager is a major actor in the query subsystem, and resides on the gateway, in the AlarmGate software. Devices are commonly added to and removed from the system, particularly in the healthcare domain where monitoring needs evolve over time. To enable the Query Manager to maintain device state, nodes register with the nearest gateway upon power-up, providing their device type, sensors, and 
hardware ID. They are assigned dynamic network IDs and localized via applicationspecific means.

The Query Manager issues background queries to devices as they are added to the network to satisfy the system's core management and tracking functionalities. Examples of background queries in AlarmNet are:

- All devices sample and report their battery supply voltage every four hours, but only if it is below $2.8 \mathrm{~V}$ (indicating imminent failure);

- Motion, tripwire, and contact-switch sensors report activations on-demand, but no more often than every $100 \mathrm{~ms}$ to debounce or dampen spurious bursts;

- Pulse oximetry devices, which are intermittently switched-on, collect heart rate and $\mathrm{SpO}_{2}$ samples every $250 \mathrm{~ms}$, but report them every $750 \mathrm{~ms}$, each an average of three samples until the device is switched off; and,

- ECG sensors immediately begin reporting a stream of raw samples every $20 \mathrm{~ms}$, using full buffering to reduce network load and energy usage.

The Query Manager is the main point of access for user interfaces, translating between higher-level query abstractions and the SenQ protocol exchanged with particular sensor devices. Connected users receive a list of active devices that is updated in real-time as registrations are received. However, most users of the system will not have detailed knowledge of its current topology. Usability is improved if they can request sensor data semantically for people and locations. This presents a few challenges for query management on the gateway.

Fig. 5 SenQ's context model. AlarmNet extensions are in grey.

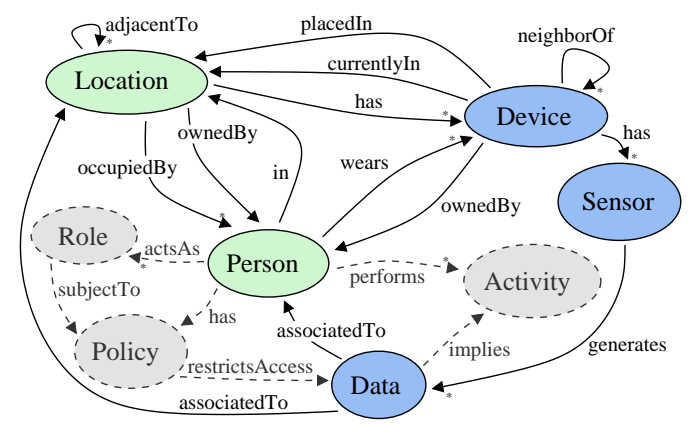

A request for sensor information about person $P$ must be mapped to a device (or group of devices) $D$ for execution. Some have static associations, such as a wearable-device owned or assigned to a user. Likewise for locations $L$ in which fixed sensor nodes are placed. But since networks for assisted-living are more humanoriented and heterogeneous than most other types of WSNs, many sensor types require dynamic binding based on a person's context (location, activity, etc). Externalizing such bindings, as in other common approaches for WSNs, results in duplicated effort to track user and device state by both the query system and the application that uses it. 
Dynamic semantic binding simplifies data access for users, but the challenge is how to provide it in a modular way that does not limit SenQ to a particular deployment environment. Our approach is to share the core of a context model, shown in Figure 5, with applications co-resident on the Query Management gateway. Relations among the core components (Device, Sensor, Location, Person, and Data) are maintained by SenQ and consulted for mapping queries to devices.

Instantiations of the system extend the model as appropriate; for example, AlarmNet adds privacy, power management, and activity analysis. These and any future extensions can access and mutate the core shared context to change SenQ's bindings of queries to sensors. AlarmNet's context-aware modules are described next, beginning with activity analysis which also interacts with the others.

\subsection{Circadian Activity Rhythms}

It is known that most people exhibit behavioral trends in the home with 24-hour cycles, are called "Circadian Activity Rhythms" (CAR). AlarmNet includes a CAR analysis program that measures the rhythmic behavioral activity of residents and detects changes within these patterns.

The CAR algorithm is statistical and predictive, and was first presented in [36]. Here we present its integration with AlarmNet and some recent extensions. CAR is used to improve both medical care and network performance. In particular, CAR supports context-aware protocols based on learned activity patterns for smart heterogeneous power management and dynamic alarm-driven privacy.

Rhythms based on an hourly distribution of the probability of user presence in every room are called presence based CAR. Those based on the density of the number of events per hour are called activity level based CAR. The CAR analysis program runs on a PC on the back-end of the system, and reads a database of resident activity.

The CAR program provides a GUI to display various information related to the activity analysis, such as the number of abnormal time periods (under-presence or over-presence in a room for presence based CAR), the degree of activity that occurred per hour and day during day or night (hypo or hyper-activity level for activity level based CAR), and the length and dates of stay of the resident.

Other graphs of the GUI display the main results of the CAR analysis. The graphs in Figure 6 present data from a clinical case study for a healthy resident who stayed 25 days in an assisted-living facility. The first one (Figure 6(a)) displays the average time the user spends in every room each hour, calculated over the number of days of the stay of the resident. On the right side, the graph indicates deviations in room presence. The experiments demonstrated that the CAR program needed a period of approximately two weeks to learn normal behavior patterns. The graphs in Figure 6(b) represent the same experiment (same subject, same clinical protocol, and same period of study), but for his activity levels.

Comparing both graphs gives complementary information concerning normal and abnormal activity levels in the different rooms of the dwelling. These graphs 

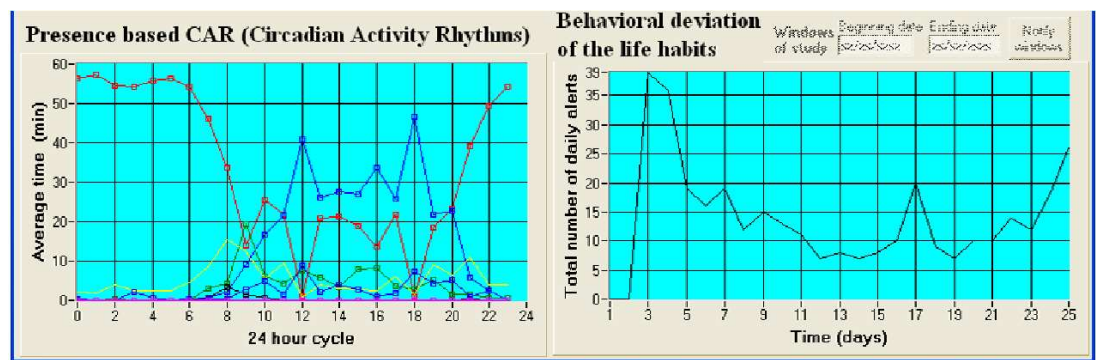

(a) Presence based CAR analysis GUI. Average time spent in every room per hour is graphed on the left side.

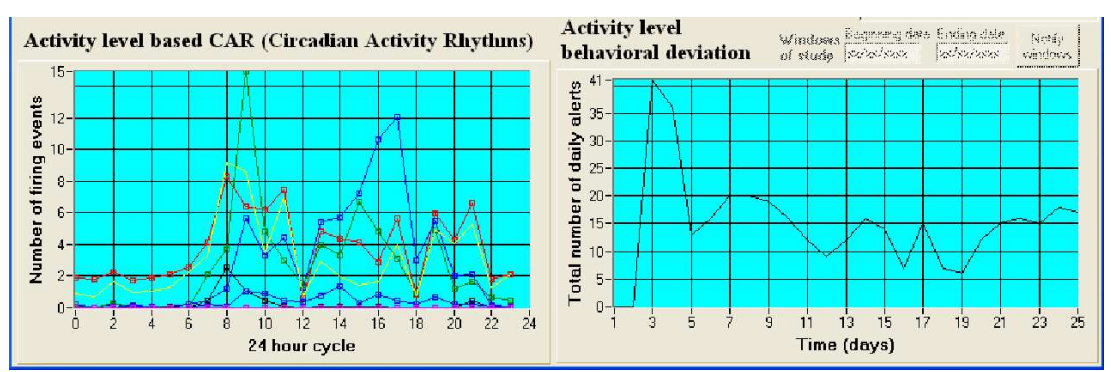

(b) Activity level based CAR analysis GUI. Average number of motion sensor events in every room per hour is on the left side.

Fig. 6 Circadian Activity Rhythm (CAR) analysis GUIs. Sums of daily deviations from the user's norm are on the right side of each GUI, showing a learning period after initial deployment.

can provide a wealth of information about activity patterns such as the sleep/wake cycle, or some medical hints to the physician about some activities of daily living (ADLs) [16] of the resident such as eating, hygiene, and sleeping. In the future, more specific ADLs will also be inferred.

After the learning period, any statistically significant deviations from learned patterns are displayed as alerts on a GUI and are sent to the AlarmGate application. Nurses or physicians can investigate the source of the trouble by focusing on the region of the anomaly as identified by CAR. The hypothesis is that deviations from a resident's own in-home behaviors can support medical diagnosis. For example, increased sleep time and multiple missed meals may indicate illness in the shortterm, or reveal overall decline in the health of the resident in the long-term.

This hypothesis was investigated clinically in collaboration with the Medical Automation Research Center (MARC) at the University of Virginia School of Medicine. Clinical behavioral patterns of older adults in assisted-living facilities were extracted from real data sets, and behavioral changes were studied by consulting the medical notebooks of the caregivers in charge of the monitored residents. The capability to detect anomalies from the norm calculated over multiple days was proved [35], but inferring pathologies or onsets of chronic pathologies remains to be explored. 


\subsection{Dynamic Context-Aware Privacy}

Data collected in AlarmNet reveals intimate details about a person's life activities and health status. As WSNs grow stronger in their capability to collect, process, and store data, personal information privacy becomes a rising concern. AlarmNet includes a framework to protect privacy and still support timely assistance to residents in critical health situations.

Emergency-aware applications demand a privacy protection framework capable of responding adaptively to each resident's health condition and privacy requirements in real-time. Traditional role-based access control, which makes access authorization decisions based on users' static roles and policies, is not flexible enough to meet this demand. A privacy protection framework was designed which is dynamically adjustable to residents' context, allows data access authorization to be evaluated at runtime, and is able to adapt to residents' health emergencies.

A key novelty is that access rulings are dynamically altered based on context inferred by the CAR and other back-end programs, when necessary. For example, if a resident has blocked access to his ECG data for nurses, but the CAR has detected serious anomalous behavior that might indicate a heart problem, then a nurse is alerted and access to the data is allowed for a period of time.

In case of an alarming health status, privacy may be restricted or relaxed depending on context and who requests the data. This decision is recorded in the database with details of the mitigating context. It can be used to notify residents later about what transpired and why, and who accessed their data during the emergency.

Other context in our system includes the resident's physiological condition (ECG, pulse, blood pressure), living environment conditions (room temperature, light levels, dust), activities, and autonomy (inferred from ADLs by the CAR). System designers may specify privacy policies at different levels of granularity, from individual sensors to residents or groups of residents.

Fig. 7 Privacy-related components in AlarmNet.

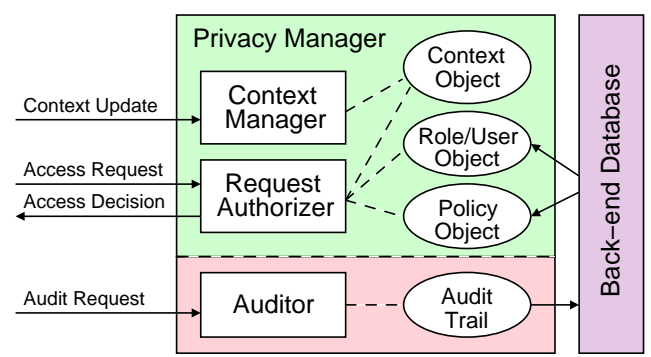

The privacy management framework resides in the AlarmGate application, and has three main functional components: the Context Manager, the Request Authorizer, and the Auditor (shown in Figure 7). 
Context Manager, as described before, maintains residents' current locations, activities, and health conditions. In the privacy module, context is indexed by the tuple $<$ context id, context subject, context value $>$.

Request Authorizer is consulted when data queries are received at the Query Manager. It makes access decisions by consulting the system's privacy policies and the context of the query subject. After each access request is decided, it is recorded by the Auditor module.

SenQ allows queries for locations and devices, which must be mapped to a resident for authorization by the Request Authorizer. Locations may be assigned an owner, such as the resident living in a particular unit. Common areas without an owner use a default subject or policy. Devices also may have an owner, for example, an ECG that is worn by a particular resident. Otherwise, the device's assigned or current location is used to determine the context subject.

Auditor maintains a trace of access requests in an audit trail, including the authorization decision made for each request (granted or denied). Dynamic decisions during alarming situations trigger the storage of details of the context for later notification of residents and investigation of misuse. Such records may be crucial in heavily regulated environments like healthcare.

\subsection{AlarmNet Summary}

AlarmNet is a wireless sensor network system designed for long-term health monitoring in assisted-living environments with two central design goals. First, system operation adapts to the individual context and behavior patterns of the residents, which feeds back to influence power management and privacy policy enforcement. Second, the system is extensible and supports a diverse collection of sensors, user interfaces, and power and privacy policies. Systems such as AlarmNet and the proliferation of individual wireless medical devices are having a transformative effect on medical care. It is being referred to as wireless medicine.

\section{Environmental Science Applications - Luster}

One of the most beneficial and interesting applications of WSNs is the ability to create a "macroscope"- to take a look at the big picture of the monitored environment. There have been many implementations of macroscopes, for example, a WSN deployed on Redwood trees [33], a wildlife monitoring site on Great Duck Island $[20,31,32]$, tracking zebras in their natural habitat [14], and monitoring volcanic eruptions [38, 37].

In this section, Luster, an environmental science application for measuring the effect of sunlight on under shrub growth on barrier islands is described. Shrub cover is increasing worldwide, most often by replacing herbaceous-dominated communities, 
especially grasslands [1]. In North America, this phenomenon has been documented in a range of environments from desert grasslands and arctic tundra to Atlantic coast barrier islands. Shrub encroachment has been linked to climate change and anthropogenic factors; however, the causes have not been thoroughly investigated. Perhaps most dramatic is the change in the light environment as community structure shifts from grassland to shrubland. Most studies measure light either at a single point in time or over periods of minutes or hours. However, there is a need to quantify light at finer temporal scales because photosynthetic responses occur within seconds and courser measurements often underestimate the effects of brief, but intense, direct light. Measuring light at a fine spatial and temporal granularity is the primary purpose of the Luster system.

Luster addresses many WSN issues including the harsh elements of nature that cause rapid device and sensor malfunction, and network links to back-end monitoring and collection systems that may be intermittent due to weather or other problems.

The main features of Luster are:

- An overlay network architecture - A hierarchical structure for sensing, communication, and storage allows replication of the system in clusters for scalability.

- Reliable, transparent, distributed storage - Fault-tolerant storage is provided by unobtrusively listening to sensor node communications, thus minimizing power requirements without the need of dedicated queries.

- Delay-tolerant networking - Access is provided to the measured data over an unreliable or intermittent network connection.

- Custom hardware - New hardware designs provide combined sensing and energy harvesting, removable storage, and lightweight in-situ WSN interfaces for deployment time validation.

- Deployment time validation - New techniques and tools were developed to increase the probability of successful deployment and long term operation of the system by looking for problems and fixing them early.

- Customizable web interface - Remote access to the WSN and visualization of sensor data is provided using reconfigurable hypertext templates.

\subsection{Luster's Architecture}

As can be inferred from the above list of capabilities, the primary challenges addressed by Luster are robust system operation, openness, and real-time. To address these challenges, Luster's architecture is composed of several layers, shown in Figure 8 . The sensor node layer is responsible for gathering, aggregating, and transmitting the measurement data. The report rate and sensor selection are configurable remotely using the SenQ sensor network query system [39]. Communication among nodes in the cluster uses LiteTDMA, a novel MAC protocol develop specifically to address the robust and real-time aspects of the system. 


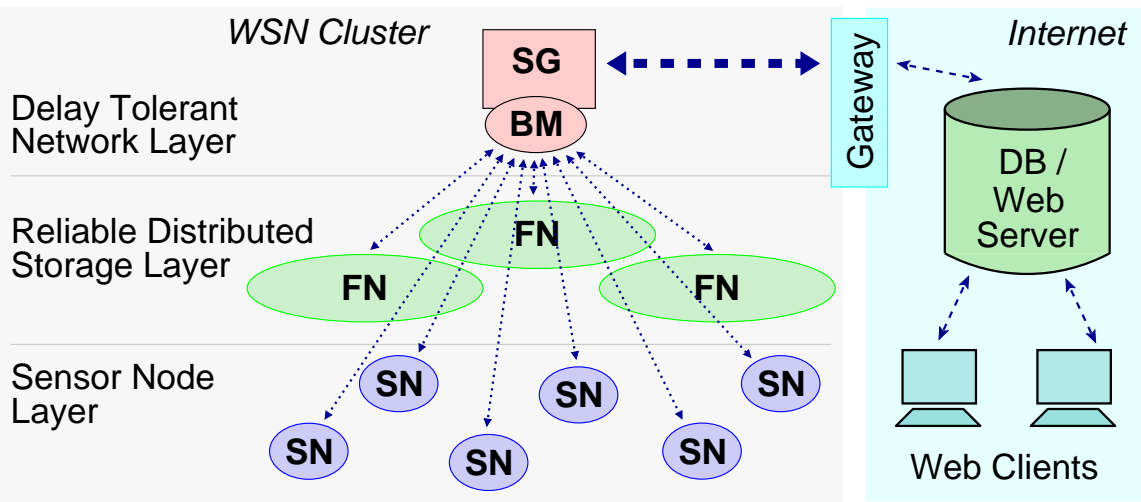

Fig. 8 Luster's hierarchical architecture.

The storage layer transparently blankets the sensor layer, collecting and filtering the data reported by sensor nodes without initiating any communication to them. Thus, bandwidth and power consumption are improved. The configuration specifies the data filtering and collection policies for each storage node, alleviating congestion internal to the storage hardware due to the flash memory delays. Reliability is provided through redundant coverage: each sensor node is monitored by at least two storage nodes. The configuration and the number of storage nodes are subject to application requirements for the fault tolerance required and cost limitations.

Above the storage layer is the delay-tolerant networking (DTN) component of the system. This consists of a base mote (denoted BM in Figure 8) attached to a stargate (SG) acting as a gateway between the IEEE 802.15.4 and IEEE 802.11 networks. Absolute reliability of this communication link is not assumed. Instead, distributed storage is used to capture all the data and either serve it in response to queries after the communication link between the WSN and the Internet is reestablished, or in a delayed fashion by collecting the removable storage cards during a subsequent visit to the deployment area. The latter is the most energy efficient method for the WSN, especially when many sensors are required.

The data monitored by the WSN is accessible over the Internet by connecting to the Luster back-end server. The server stores the incoming WSN data stream to a database and also issues data queries to the WSN as a DTN component detects losses.

Using a web browser, a user can request and view historical data as well as the last captured "almost real-time" data from the back-end server. Web pages are generated from HTML templates, in which the WSN data, including the sensor readings, are embedded in the HTML as custom tags. This allows for a user-centric customizable web interface that is specific to the chosen application.

An example of an application-neutral display is shown in Figure 9, in which a node's current sensor readings have been requested. Values from the eight ADC channels are tabulated and graphed. Using custom HTML tags, a user can add semantically meaningful labels for the channels and their values. Near real-time dis- 


Timestamp: Mon Apr $1617: 49: 042007$
\begin{tabular}{|c|c|c|c|}
\hline ADC & Value & $0 \%$ & $50 \%$ \\
\hline 1 & 792 & & \\
\hline 2 & 1 &. & \\
\hline 3 & 762 &. & \\
\hline 4 & 851 &. & \\
\hline 5 & 793 &. \\
\hline 6 & 831 &. \\
\hline 7 & 814 & \\
\hline 8 & 512 & \\
\hline
\end{tabular}

Fig. 9 Luster web server generated page reporting ADC levels for a pre-selected remote sensor node.

play of sensor readings supports online diagnostics to determine, for example, that ADC channel two in Figure 9 is faulty and stuck at a low value.

For scalability, the WSN architecture described (and shown on the left side of Figure 8 ) is replicated into multiple clusters that cover the entire deployment area. Clusters use single-hop communication internally, and are adjoined and managed by the stargate node using several techniques.

First, each cluster is assigned a communication channel that avoids or minimizes interference with its neighbors. One challenge with a multiple channel approach is the likelihood of the base mote missing communications on one channel while listening to another. This challenge is met by the delay-tolerant design of Luster, which operates even when communications from the WSN clusters to the main back-end server are lost. Distributed storage and delay-tolerant networking components allow recovery of data as described above.

A second approach for inter-cluster interference minimization is to interleave the LiteTDMA MAC communication schedules so that when one cluster is communicating, the others are asleep. The stargate node acts as a super-master to coordinate the schedules. Finally, the transmission power of nodes can be adjusted to match the cluster's coverage area.

\subsection{Luster Summary}

Many environmental science applications have been implemented with WSN technology. Many of them have similar requirements to Luster such as remote access, reliable data collection and storage, and real-time viewing. There is often a need for a specialized network, e.g., in Luster the coverage area for each 124 sensors is on the order of meters allowing a single hop local network based on a TDMA access control protocol. In other cases, e.g., Zebranet, there is a requirement to support 
mobility. In the future, as the underlying WSN technology matures, there should be more emphasis placed on creating knowledge from the data.

\section{Summary}

Wireless sensor networks are entering a second generation. The first generation has seen many new research challenges being defined and solved. This has resulted in many interesting systems being implemented. Three of these systems, VigilNet (for military surveillance), AlarmNet (home health care and assisted living), and Luster (environmental science) are described in this Chapter as representative of this first generation. New challenges have emerged for second generation wireless sensor networks including: creating knowledge from raw data, robust system operation, dealing with openness and heterogeneity, security, privacy, real-time, and control and actuation. This is not a complete list of challenges, but these do constitute some of the major challenges as wireless sensor networks become widespread and move into many other application domains such as agriculture, energy, and transportation.

\section{Acknowledgements}

We thank all the students that worked on one or more of these systems. The work was supported, in part, by NSF grants CNS-0614870, CNS-0614773, CNS0626616, CNS-0626632, the DARPA NEST project and a DoD MURI.

\section{References}

1. Brantley, S.T., Young, D.R.: Leaf-area index and light attenuation in rapidly expanding shrub thickets. Ecology 88(2), 524-530 (2007)

2. Cao, Q., Stankovic, J.A.: An in-field maintenance framework for wireless sensor networks. In: Proc. of DCOSS (2008)

3. Capkun, S., Hubaux, J.P.: Secure positioning of wireless devices with application to sensor networks. In: Proceedings of IEEE INFOCOM (2005)

4. Cerpa, A., Estrin, D.: Ascent: Adaptive self-configuring sensor networks topologies. In: Proceedings of INFOCOM (2002)

5. Chan, H., Perrig, A., Song, D.: Random key predistribution schemes for sensor networks. In: IEEE Symposium on Research in Security and Privacy, pp. 197-213 (2003)

6. CrossBow: Mica2 data sheet. Available at http: //www. xbow. com

7. Dutta, P., Grimmer, M., Arora, A., Biby, S., Culler, D.: Design of a Wireless Sensor Network Platform for Detecting Rare, Random, and Ephemeral Events. In: IPSN'05 (2005)

8. Ganti, R.K., Jayachandran, P., Abdelzaher, T.F.: SATIRE: A software architecture for Smart AtTIRE. In: 4th ACM Conference on Mobile Systems, Applications, and Services (Mobisys 2006) (2006)

9. Gu, L., Stankovic, J.A.: t-kernel: Providing reliable os support for wireless sensor networks. In: Proc. of ACM Conf. on Embedded Networked Sensor Systems (SenSys’06) (2006) 
10. He, T., Krishnamurthy, S., Yan, L.L.T., Gu, L., Stoleru, R., Zhou, G., Cao, Q., Vicaire, P., Stankovic, J., Abdelzaher, T., Hui, J., Krogh, B.: Vigilnet: An integrated sensor network system for energy efficient surveillance. ACM Transactions on Sensor Networks 2(1), 1-38 (2006)

11. He, T., Vicaire, P., Yan, T., Cao, Q., Zhou, G., Gu, L., Luo, L., Stoleru, R., Stankovic, J.A., Abdelzaher, T.: Achieving Long-Term Surveillance in VigilNet. In: The 25th Conference on Computer Communications (INFOCOM 2006), pp. 1-12 (2006)

12. He, T., Vicaire, P., Yan, T., Luo, L., Gu, L., Zhou, G., Stoleru, R., Cao, Q., Stankovic, J., Abdelzaher, T.: Achieving real-time target tracking using wireless sensor networks. ACM Transactions on Embedded Computing System 30(5), 627-633 (2008)

13. Hui, J., Culler, D.: The Dynamic Behavior of a Data Dissemination Protocol for Network Programming at Scale. In: Second ACM Conference on Embedded Networked Sensor Systems (SenSys 2004) (2004)

14. Juang, P., Oki, H., Wang, Y., Martonosi, M., Peh, L.S., Rubenstein, D.: Energy-efficient computing for wildlife tracking: design tradeoffs and early experiences with zebranet. In: Proc. of ASPLOS-X, pp. 96-107 (2002)

15. Kamat, P., Zhang, Y., Trappe, W., Osturk, C.: Enhancing source location privacy in sensor network routing. In: Proc. Int. Conf. on Distributed Computing Systems (2005)

16. Katz, S., Ford, A.B., Moskowitz, R.W., Jackson, B.A., Jaffe, M.W.: Studies of illness in the aged. the index of adl: A standardized measure of biological and psychosocial function. Journal of the American Medical Association 185, 914-919 (1963)

17. Luo, L., Abdelzaher, T., He, T., Stankovic., J.A.: EnviroSuite: An Environmentally Immersive Programming Framework for Sensor Networks. ACM Transactions on Embedded Computing System, Special issue on Dynamically Adaptable Embedded Systems 5(3) (2006)

18. Luo, L., He, T., Zhou, G., Gu, L., Abdelzaher, T., Stankovic, J.: Achieving Repeatability of Asynchronous Events in Wireless Sensor Networks with EnviroLog. In: The 25th Conference on Computer Communications (INFOCOM 2006), pp. 1-14 (2006)

19. Madden, S., Franklin, M.J., Hellerstein, J.M., Hong, W.: TinyDB: An acqusitional query processing system for sensor networks. ACM TODS 30(1), 122-173 (2005)

20. Mainwaring, A., Culler, D., Polastre, J., Szewczyk, R., Anderson, J.: Wireless sensor networks for habitat monitoring. In: Proc. of WSNA, pp. 88-97 (2002)

21. Maroti, M., Kusy, B., Simon, G., Ledeczi, A.: The Flooding Time Synchronization Protocol. In: Second ACM Conference on Embedded Networked Sensor Systems (SenSys 2004) (2004)

22. Paradis, L., Han, Q.: A survey of fault management in wireless sensor networks. Journal of Network and Systems Management 15(2) (2007)

23. Perrig, A., Stankovic, J., Wagner, D.: Security in wireless sensor networks. Commun. ACM 47(6), 53-57 (2004)

24. President's Information Technology Advisory Committee (PITAC), Revolutionizing Health Care Through Information Technology (2004). URL: http://www.nitrd.gov/ pitac/reports/20040721_hit_report.pdf

25. Ramanathan, N., Chang, K., Girod, L., Kapur, R., Kohler, E., Estrin, D.: Sympathy for the sensor network debugger. In: In Proceedings of the 3rd ACM International Conference on Embedded Networked Sensor Systems (SenSys) (2005)

26. Rost, S., Balakrishnan, H.: Memento: A health monitoring system for wireless sensor networks. In: Proceedings of IEEE SECON (2006)

27. Selavo, L., Wood, A., Cao, Q., Srinivasan, A., Liu, H., Sookoor, T., Stankovic, J.: Luster: Wireless sensor network for environmental research. ACM Sensys (2007)

28. Selavo, L., Zhou, G., Stankovic, J.: SeeMote: In-situ visualization and logging device for wireless sensor networks. In: Proc. of BASENETS (2006)

29. Stankovic, J.: When sensor and actuator networks cover the world. ETRI Journal 30(5), 627$633(2008)$

30. Stoleru, R., He, T., Stankovic, J.A.: Walking GPS: A Practical Solution for Localization in Manually Deployed Wireless Sensor Networks. In: 1st IEEE Workshop on Embedded Networked Sensors EmNetS-I (2004) 
31. Szewczyk, R., Mainwaring, A., Polastre, J., Anderson, J., Culler, D.: An analysis of a large scale habitat monitoring application. In: Proc. of SenSys, pp. 214-226 (2004)

32. Szewczyk, R., Polastre, J., Mainwaring, A.M., Culler, D.E.: Lessons from a sensor network expedition. In: H. Karl, A. Willig, A. Wolisz (eds.) EWSN, Lecture Notes in Computer Science, vol. 2920, pp. 307-322. Springer (2004)

33. Tolle, G., Polastre, J., Szewczyk, R., Culler, D., Turner, N., Tu, K., Burgess, S., Dawson, T., Buonadonna, P., Gay, D., Hong, W.: A macroscope in the redwoods. In: Proc. of SenSys, pp. 51-63 (2005)

34. Vicaire, P., He, T., Yan, T., Cao, Q., Gu, G.Z.L., Luo, L., Stoleru, R., Stankovic, J., Abdelzaher, T.: Achieving long term surveillance in vigilnet. IEEE Computer 5(1), 1-39 (2009)

35. Virone, G., Alwan, M., Dalal, S., Kell, S., Turner, B., Stankovic, J.A., Felder, R.: Behavioral patterns of older adults in assisted living. Information Technology in Biomedicine 12(3), 387-398 (2008)

36. Virone, G., Noury, N., Demongeot, J.: A system for automatic measurement of circadian activity deviation in telemedicine. IEEE Transactions on Biomedical Engineering 49(12), 14631469 (2002)

37. Welsh, M., Werner-Allen, G., Lorincz, K., Marcillo, O., Johnson, J., Ruiz, M., Lees, J.: Sensor networks for high-resolution monitoring of volcanic activity. In: Proc. of SOSP, pp. 1-13 (2005)

38. Werner-Allen, G., Lorincz, K., Welsh, M., Marcillo, O., Johnson, J., Ruiz, M., Lees, J.: Deploying a wireless sensor network on an active volcano. IEEE Internet Computing 10(2), $18-25$ (2006)

39. Wood, A., Stankovic, J., Virone, G., Selavo, L., He, Z., Cao, Q., Doan, T., Wu, Y., Fang, L., Stoleru, R.: Context-aware wireless sensor networks for assisted living and residential monitoring. IEEE Network 22(4), 26-33 (2008)

40. Wood, A.D., Selavo, L., Stankovic, J.A.: SenQ: An embedded query system for streaming data in heterogeneous interactive wireless sensor networks. In: International Conference on Distributed Computing in Sensor Systems (DCOSS), Lecture Notes in Computer Science, vol. 5067, pp. 531-543. Springer Berlin (2008)

41. Wood, A.D., Stankovic, J.A.: Denial of service in sensor networks. IEEE Computer 35(10), 54-62 (2002)

42. Zhou, G., He, T., Stankovic, J.A.: Impact of Radio Irregularity on Wireless Sensor Networks. In: The Second International Conference on Mobile Systems, Applications, and Services (MobiSys) (2004) 